\title{
Fluid Electric Force Microscopy for Charge Density Mapping in Biological Systems
}

\author{
Amber S. J ohnson, ${ }^{\dagger}$ Colleen L. Nehl, ${ }^{\ddagger}$ Monica G. Mason, ${ }^{\ddagger}$ and \\ J ason H. Hafner*,‡,§
}

Departments of Electrical and Computer Engineering, Physics and Astronomy, and Chemistry, Rice University, 6100 Main Street, Houston, Texas 77005

Received J uly 11, 2003. In Final Form: October 6, 2003

\begin{abstract}
Electric force microscopy has been adapted for mapping surface charge density in aqueous solutions. The electric double layer interaction between the tip and the sample provides a contrast mechanism sensitive only to the surface charge density as other parameters are held constant. The resulting charge maps are acquired at typical atomic force microscopy scanning rates and approximately 25-nm resolution. The contrast is well-described by a simpleexpressi on for thetip-sampledoublelayer interaction in electrolyte solutions. Fluid electric force microscopy is highly sensitive and nondestructive, as demonstrated with charge density maps of fluid-phase-supported bilayer membranes and single DNA molecules.
\end{abstract}

\section{Introduction}

The atomic force microscope (AFM) has sufficient force sensitivity to measurescreened el ectrostaticinteractions between the tip and the sample in aqueous electrolyte solutions. Early investigations demonstrated that tipsample forces are well-described by electric double layer interactions, van der Waals interactions, and hydration forces over a broad range of $\mathrm{pH}$ values, ionic strengths, and tip-sample separations. ${ }^{1}$ Beginning with a formula derived from thePoisson-Boltzmann equation for charged planar surfaces, ${ }^{2}$ an approximation for the tip-sample force, $F$, as a function of the separation, $L$, was cal culated: ${ }^{3}$

$$
F(L)=\frac{4 \pi \lambda r \sigma_{\mathrm{s}} \sigma_{\mathrm{t}}}{\epsilon \epsilon_{0}} \mathrm{e}^{-\mathrm{L} / \lambda}
$$

where $r$ is the tip radius, $\lambda$ is the Debye length, $\epsilon$ is the dielectric constant of the medium, $\epsilon_{0}$ is the vacuum permittivity, and $\sigma_{\mathrm{t}}$ and $\sigma_{\mathrm{s}}$ are the tip and sample charge densities, respectively. This approximation is valid if the tip radius and tip-sample separation are greater than the Debye length. As an imaging tool, this el ectric double layer interaction offers a signal that depends only on the sample's surface charge density, assuming the tip properties and the tip-sample separation can be held constant. Two such charge density mapping techniques based on the AFM have already been demonstrated. The first empl oys contact-mode imaging, in which a feedback loop adjusts thesamplez position to keep thetip-sample force at a constant set-point value. By carefully adjusting the set point for weak repulsive or attractive forces, double layer interactions can contributeto theimage contrast. ${ }^{4-7}$

* Towhom correspondenceshould beaddressed. E-mail: hafner@ rice.edu.

${ }^{\dagger}$ Department of Electrical and Computer Engineering, Rice University.

‡ Department of Physics and Astronomy, Rice University.

$\S$ Department of Chemistry, Rice University.

(1) Butt, H.-J . Biophys. J . 1991, 60, 1438-1444

(2) Parsegian, V. A. Gingell, D. Biophys. J 1972, 12, 1192-1204.

(3) Butt, H.-J . Biophys. J . 1991, 60, 777-785.

(4) Rotsch, C.; Radmacher, M. Langmuir 1997, 13, 2825-2832.

(5) Manne, S.; Cleveland, J. P.; Gaub, H. E.; Stucky, G. D.; Hansma,

P. K. Langmuir 1994, 10, 4409-4413.

(6) Senden, T. J .; Drummond, C. J .; Kekicheff, P. Langmuir 1994, $10,358-362$.

(7) Muller, D. J .; Engel, A. Biophys. J . 1997, 73, 1633-1644.
The contact mode can also map the charge density by subtracting the images recorded at different salt concentrations to reveal the electrostatic contribution to the image contrast. ${ }^{8}$ The second method relies on forcecurves in which the tip-sample force is measured as a function of the tip-sample separation. F orce curves are recorded over an array of points on the sample, referred to as a "force-volume" measurement. Each curve can then be analyzed to determine several parameters, including the sampleheight, adhesion force, and samplechargedensity, as a function of the position. $4,9,10$

The ability to map charge density in aqueous solutions at the nanometer scale could havea si gnificant impact on many areas of science and technology. Contact-mode imaging has provided subnanometer resolution on the structureand chargedensity of certain biological samples such as ordered membrane proteins. ${ }^{8}$ Force-volume measurements have elucidated the adsorption behavior of hemimicelles on hydrophobic substrates ${ }^{5}$ as well as charge heterogeneity on colloid supports. ${ }^{11} \mathrm{H}$ owever, these methods havecertain limitations. The contact moderesults in a convolution of topographic and electrostatic sample information. Although it can be removed by image subtraction, this precludes studies of systems sensitiveto ionicstrength. Also, thermal drift in thecantilever requires peri odic manual adjustments, hindering long-term measurements. ${ }^{4-6}$ F orce- volume measurements avoid topographic and electrostatic convolution, but compared to typical AFM imaging conditions they are slow and have limited pixel resolution, typically $64 \times 64$ pixels or less. As with any microscopy, surface charge mapping would benefit from improved resolution, imaging speed, contrast, and versatility.

We have adapted electric force microscopy $(\mathrm{EFM})^{12}$ for measurements of electric double layer interactions in aqueous solutions. In somemanifestations, EFM employs the "lift mode" in which the tip scans each line in the imagetwice(Figure1). In thefirst scan, the AFM measures

(8) Philippsen, A.; Im, W. P.; Engel, A.; Schirmer, T.; Roux, B.; Muller, D. J. Biophys. J . 2002, 82, 1667-1676.

(9) Radmacher, M.; Cleveland, J. P.; Fritz, M.; Hansma, H. G.; Hansma, P. K. Biophys. J . 1994, 66, 2159-2165.

(10) Heinz, W. F.; Hoh, J . H. Biophys. J . 1999, 76, 528-538.

(11) Shellenberger, K.; Logan, B. E. Environ. Sci. Technol. 2002, 36, 184-189.

(12) Martin, Y.; Abraham, D. W.; Wickramasinghe, H. K. Appl. Phys. Lett. 1988, 52, 1103-1105. 


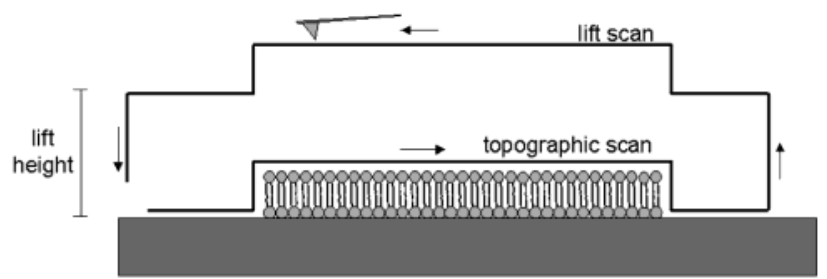

Figure 1. Tip path in the lift mode over a supported lipid bilayer. The actual tip path includes a full trace and retrace for both the topographic and the lift scans. The figure shows only a topographic trace and a lift retrace for clarity.

thesampletopography in thetapping mode. ${ }^{13}$ In thesecond scan, thetip lifts off the surface a set amount and repeats the measured topography at a constant tip-sample separation. Electrostatic forcegradients aremeasured on the lift scan through resonance shifts in stiff silicon cantilevers. A similar method could be applied to fluid imaging; however, theelectric doublelayer forces and force gradients are much smaller and the cantilever is critically damped, ${ }^{14}$ so the alternating current (AC) measurements are not as sensitive. Instead, we have used softer silicon nitride cantilevers and measured their static deflection during the lift scan, therefore directly measuring the double layer force at a constant tip-sample separation (see Figure 1). Because the cantilever acts like a simple spring for small deflections, the deflection signal varies linearly with the doublelayer forceand, therefore, linearly with differences in the sample charge density.

\section{Experimental Methods}

Supported Li pi d Bilayers. 1,2-Diol eoyl-3-trimethylammoniumpropane (DOTAP), 1,2-dioleoyl-sn-glycero-3-phosphocholine (DOPC), and 1,2-diol eoyl-sn-glycero-3-[phospho-L-serine] sodium salt (DOPS) were purchased lyophilized from Avanti Polar Lipids (Alabaster, AL). The DOPS vesi des were prepared by vortexing a $1 \mathrm{mg} / \mathrm{mL}$ solution of the lyophilized powder in deionized (DI) water for $30 \mathrm{~min}$. Cationic vesicles were prepared by drying down a $5 \mathrm{mg} / \mathrm{mL}$ chloroform solution of a 4:1 mole ratio of DOTAP/ DOPC in a rotary evaporator under a rough vacuum rotating at $200 \mathrm{rpm}$ for $30 \mathrm{~min}$. The film was then hydrated with DI water, still rotating, for $30 \mathrm{~min}$. Small unilamellar vesicles (SUVs) were created from each solution by extrusion through a 100-nm polycarbonatefilter (MiniExtruder, Avanti). Tocreatesupported bilayers for AFM imaging, $30 \mu \mathrm{L}$ of the SUV solutions were incubated for $20 \mathrm{~min}$ over freshly cleaved mica for cationiclipids or poly-L-lysine-coated mica for DOPS. The samples were then dipped in $50 \mathrm{~mL}$ of the electrolyte solution ( 1 or $3 \mathrm{mM} \mathrm{NaCl}$ ) to rinse the surface, and the mica was transferred to the AFM without drying.

DNA. $\lambda$-DNA was purchased from New England BioLabs (Beverly, MA) and diluted to $500 \mathrm{ng} / \mathrm{mL}$ in $40 \mathrm{mM}$ Tris buffer, $\mathrm{pH}$ 7.4. F reshly cleaved mica was treated with $0.1 \%$ poly-L-lysine for 5 min then rinsed with DI water. A total of $20 \mu \mathrm{L}$ of the DNA dilution was placed on the mica. After $20 \mathrm{~min}$, the mica was then transferred to the AFM without rinsing. For fluid electric force microscopy (FEFM), the solution was further diluted by exchanging with $30 \mu \mathrm{L}$ of DI water three times. We estimate this "rinsing" drops the final Tris concentration to $2.6 \mathrm{mM}$

AFM. All measurements were carried out with a Multimode AFM and Nanoscope IV controller (Veeco, Santa Barbara, CA). Topographic images were acquired in the fluid tapping mode using silicon nitridecantilevers (NP, nominally 0.12 or $0.06 \mathrm{~N} / \mathrm{m}$, Veeco) driven at a tuning peak of $30 \mathrm{kHz}$ (most likely not the cantilever resonance). Thelift modewas performed as a standard function of the Nanoscopesoftwareversion 5.12r3. Tip oscillations were turned off during the lift scan, and the tip deflection was

(13) Zhong, Q.; Inniss, D.; Kjoller, K.; Elings, V. B. Surf. Sci. Lett. 1993, 290, L688-L692.

(14) Lantz, M.; Liu, Y. Z.; Cui, X. D.; Tokumoto, H.; Lindsay, S. M. Surf. Interface Anal. 1999, 27, 354-360. taken directly from the split photodiode difference signal via a signal access module and read as an auxiliary input channel. Thetopographicand deflection signals werefirst-order flattened with stop bands over thelipid regions. Data for the pl ots in Figure 3 weretaken by averaging the deflection valuefrom many pixels (typically several hundred) over the lipids and the mica regions and subtracting the averages.

\section{Results}

Figure 2 shows a FEFM image of a mica-supported DOTAP/DOPC membrane imaged in $3 \mathrm{mM} \mathrm{NaCl}$ with a lift height of $20 \mathrm{~nm}$. The lift scan (right) d early shows a downward deflection of the cantilever over the lipid membrane, corresponding to an attractive double layer interaction between thenegatively charged silicon nitride ti $p^{15}$ and the positively charged cationic lipid headgroups. Theresolution in thechargedensity map can beestimated from the width of the step in the FEFM contrast at the edge of the supported membrane. The observed full width in Figure 2 is approximately $25 \mathrm{~nm}$, a factor of 2 improvement over the force-vol umemeasurements. ${ }^{4}$ The FEFM contrast was measured as a function of the lift height, analogous to traditional force-curve measurements. Three such data sets are shown in Figure 3, including both attractive (DOTAP/DOPC) and repulsive (DOPS) interactions. The exponential fits yield Debye lengths, given in Table 1, in reasonable agreement with the values calculated by $\lambda=0.304 \mathrm{~nm} / \sqrt{ } \mathrm{C}$, where $C$ is the monovalent salt concentration. To confirm that there is notopographiccontribution tothedeflection signal, FEFM measurements were carried out on a roughened mica surface that contains topography but no charge density variations. Figure 4 demonstrates the lack of FEFM contrast on this sample when imaged in $1 \mathrm{mM} \mathrm{NaCl}$ with a 10-nm lift height. Figure 5 displays theFEFM of a region of $\lambda$-DNA deposited on poly-L-lysine-treated mica and imaged in $2.6 \mathrm{mM}$ Tris buffer. The deflection signal indicates a repulsive interaction consistent with the negativecharge density of $\lambda$-DNA as a result of ionization of the phosphate backbone.

\section{Discussion}

FEFM provides a map of the sample charge density based on the electric double layer interaction defined in eq 1. For a precise description of the FEFM contrast, consider the two regions labeled $A$ and $B$ in Figure 2. The tip deflection, $d$, on the lift scan in these regions will be

$$
\begin{aligned}
& \mathrm{d}_{\mathrm{A}}=\frac{\mathrm{F}_{\mathrm{A}}}{\mathrm{k}}=\sigma_{\mathrm{A}} \frac{4 \pi \lambda \mathrm{r} \sigma_{\mathrm{t}}}{\mathrm{k} \epsilon \epsilon_{0}} \mathrm{e}^{-\mathrm{L} / \lambda} \\
& \mathrm{d}_{\mathrm{B}}=\frac{\mathrm{F}_{\mathrm{B}}}{\mathrm{k}}=\sigma_{\mathrm{B}} \frac{4 \pi \lambda r \sigma_{\mathrm{t}}}{\mathrm{k} \epsilon \epsilon_{0}} \mathrm{e}^{-\mathrm{L} / \lambda}
\end{aligned}
$$

where $\mathrm{F}$ is the double layer interaction force between the tip and the sample, $\mathrm{k}$ is the cantilever spring constant, and $L$ is the lift height. The lift image contrast is due to changes in deflection:

$$
\Delta \mathrm{d}=\mathrm{d}_{\mathrm{B}}-\mathrm{d}_{\mathrm{A}}=\left(\sigma_{\mathrm{B}}-\sigma_{\mathrm{A}}\right) \frac{4 \pi \lambda \mathrm{r} \sigma_{\mathrm{t}}}{\mathrm{k} \epsilon \epsilon_{0}} \mathrm{e}^{-\mathrm{L} / \lambda}
$$

If the deflection signal over the mica substrate is arbitrarily assigned as 0 , the image contrast has a linear dependence on the difference between the bilayer surface charge density and the mica surface charge density.

(15) Senden, T.J .; Drummond, C. J . Colloids Surf. 1995, 94, 29-51. 

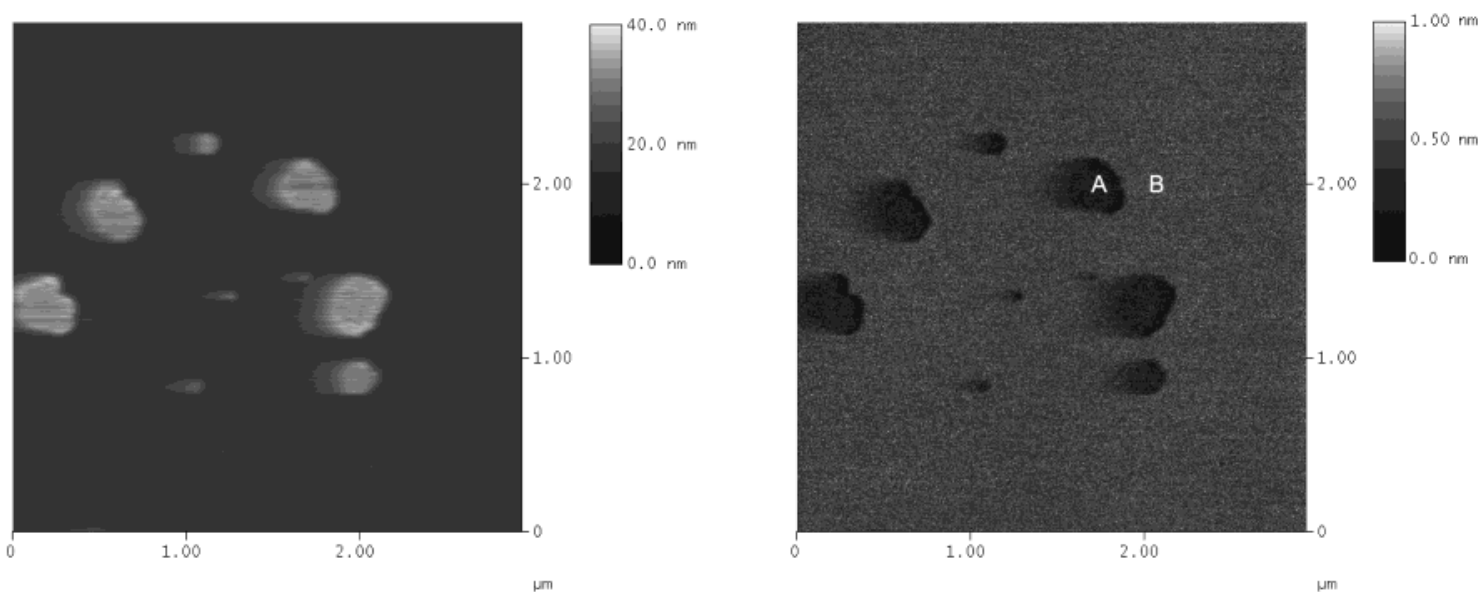

Figure 2. Topography (left) and lift mode deflection (right) of a mica-supported DOTAP/DOPC bilayer in $3 \mathrm{mM} \mathrm{NaCl}$. Downward deflections (darker contrast) imply regions of more positive charge density.

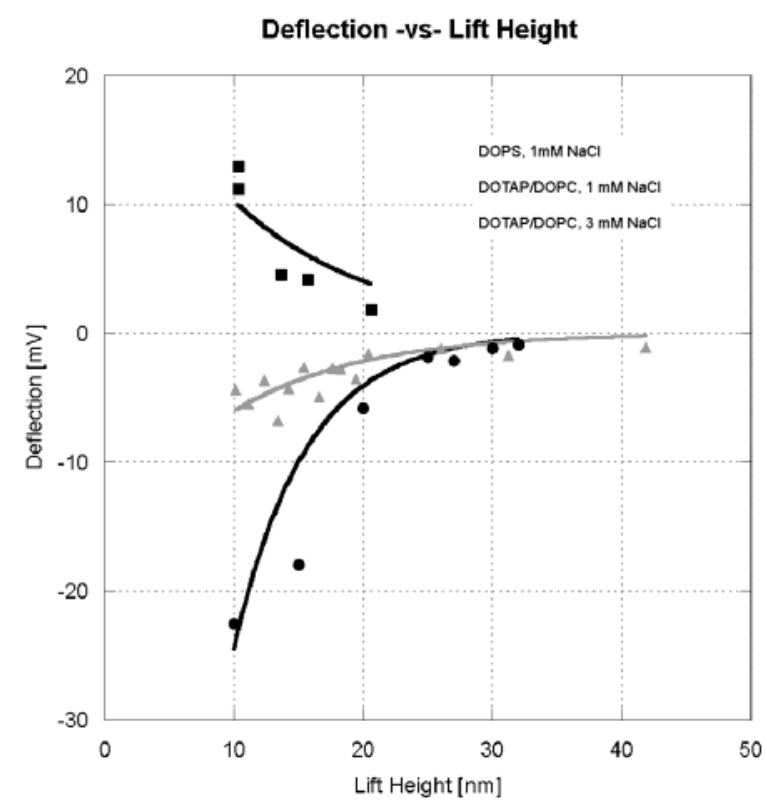

Figure 3. Lift mode deflection plotted as a function of the lift height. These plots are similar to traditional force curves and yield reasonable Debye lengths (see Table 1).

Table 1. Measured Debye Lengths from the Fits in Figure 3, as Well as the Expected Debye Length Based on the Electrolyte Concentration

\begin{tabular}{lccc}
\hline \multicolumn{1}{c}{ lipid } & electrolyte & $\begin{array}{c}\text { measured Debye } \\
\text { length [nm] }\end{array}$ & $\begin{array}{c}\text { calculated Debye } \\
\text { length [nm] }\end{array}$ \\
\hline DOPS & $1 \mathrm{mM} \mathrm{NaCl}$ & $11 \pm 7$ & 9.6 \\
DOTAP/DOPC & $1 \mathrm{mM} \mathrm{NaCl}$ & $10 \pm 3$ & 9.6 \\
DOTAP/DOPC & $3 \mathrm{mM} \mathrm{NaCl}$ & $6 \pm 2$ & 5.6
\end{tabular}

We conclude that eq 2 accuratel y describes the contrast in FEFM on the basis of several observations. First, the correct interaction polarity is always observed: the negatively charged silicon nitride tip yields repulsive interactions over the negatively charged DOPS bilayers and DNA and attractive interactions over the positively charged DOTAP/DOPC bilayers. Second, the decay length of theFEFM signal matches the el ectrolyteDebyelength, as predicted by eq 1 and demonstrated in Table 1 . Finally, the measured prefactor of eq 2 is of the correct order of magnitude. Using the nominal tip radius (30 nm), cantilever stiffness $(0.06 \mathrm{~N} / \mathrm{m})$, and $0.01 \mathrm{C} / \mathrm{m}^{2}$ for thecharge density magnitudes, ${ }^{6,16}$ eq 2 yiel ds an expected deflection

(16) Butt, H.-J . Biophys. J . 1992, 63, 578-582. of approximately $0.3 \mathrm{~nm}$ for a 20 -nm lift height, similar tothat shown in Figure2. Notethat these assumed values can vary substantially between tips and may not be wellcharacterized. Therefore, FE FM consistently provides only a qualitative measure of the sample charge density. An accurate, quantitative measurement could be achieved but would requireknowledge of thetip radius and surface charge, as well as a measure of the absolute cantilever deflection or a reference surface of known charge density. Also note the assumption that tapping-mode topography leads to a constant tip-sample separation during the lift scan. I naccurateheight measurements dueto doublelayer forces ${ }^{7}$ or sample compression ${ }^{17}$ will invalidate this assumption and alter the charge density map. For biomolecular imaging, such errors are usually small compared tothelift height under typical FEFM conditions, especially for the carefully controlled fluid tapping mode. ${ }^{18,19} \mathrm{For}$ example, a 1-nm topographic error in a solution with a Debye length of $10 \mathrm{~nm}$ and a lift height of $20 \mathrm{~nm}$ only affects the electric double layer force by $10 \%$. In addition, only relative compression is an issue because only relative charge density is measured. If the tip compresses an entire soft sample evenly, it will simply subtract a constant factor from the charge density map.

FEFM has two central advantages over contact-mode and force-volume techniques for mapping the surface charge in solution. FEFM generates high-resolution topography and charge maps at typical AFM imaging rates. The current experiments typically measured $256 \times$ 256 pixels at $2 \mathrm{~Hz}$ (1 line/s in the lift mode), which corresponds to an image every $4.3 \mathrm{~min}$, as opposed to 1 $\mathrm{h}$ of acquisition for $64 \times 64$ pixel images with forcevolume. ${ }^{10}$ While the contact mode can generate images somewhat faster, it is subject to thermal drift in the cantilever that can obscure the contrast. 4,5,15 Because tapping-mode AFM and the lift scan trajectory are based on AC cantilever feedback, they are relatively immuneto thermal drift; that is, it is small on the time scale of a single scan line. In addition, FEFM provides a charge map that is independent of topography, without the need for multiple scans under varying electrolyte conditions. ${ }^{8}$

FEFM is similar to chemical force microscopy ${ }^{20}$ (CFM) in that it provides contrast on the basis of the chemical

(17) Mou, J .; Czajkowsky, D. M.; Zhang, Y.; Shao, Z. FEBS Lett. 1995, 371, 279-282.

(18) Schabert, F. A.; Rabe, J . P. Biophys. J . 1996, 70, 1514-1520.

(19) Moller, C.; Allen, M.; Elings, V. B.; Engel, A.; Muller, D. J . Biophys. J. 1999, 77, 1150-1158.

(20) Frisbie, C. D.; Rozsnyai, L. F.; Noy, A.; Wrighton, M. S.; Lieber, C. M. Science 1994, 265, 2071-2074. 

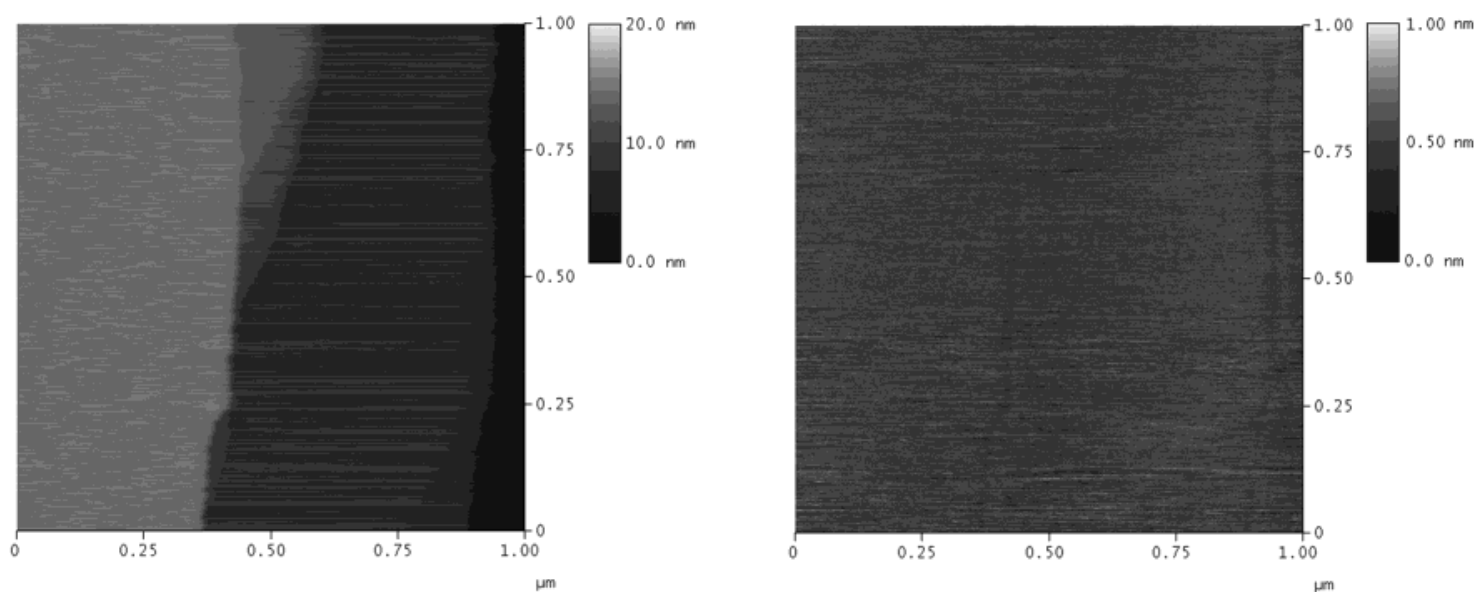

Figure 4. Topography (left) and lift mode deflection (right) on mica steps with a 20-nm lift height in $1 \mathrm{mM} \mathrm{NaCl}$.
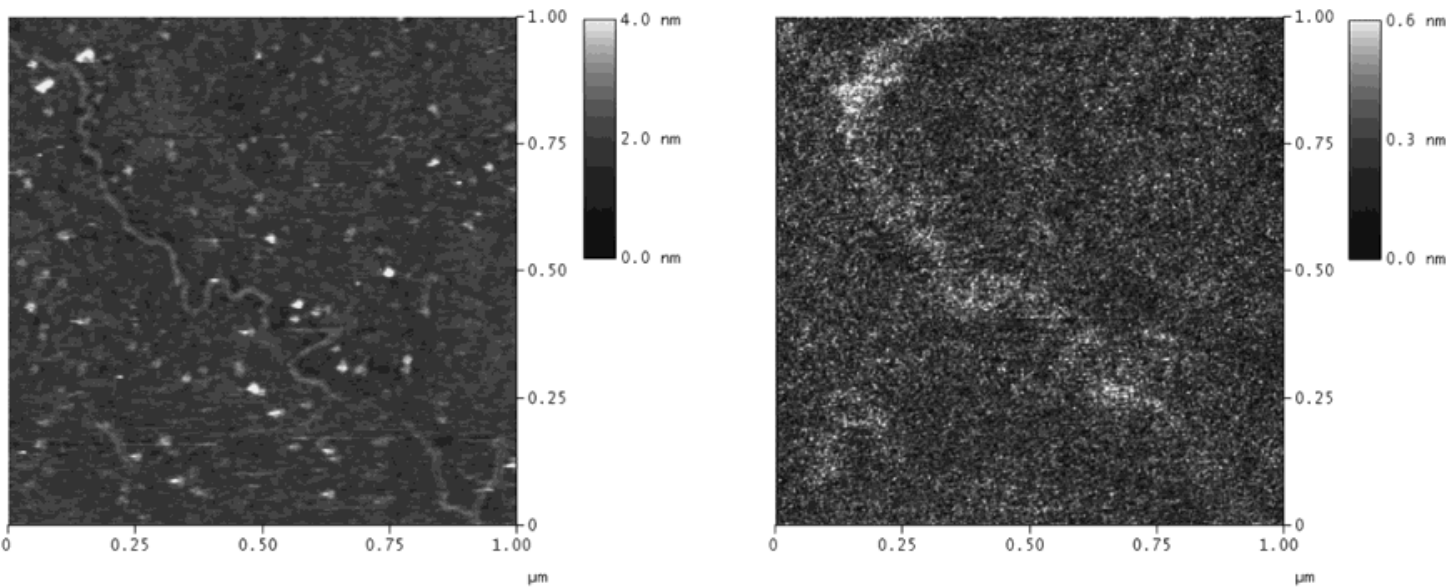

Figure 5. Topography (left) and lift mode deflection (right) of $\lambda$-DNA on poly-L-lysine-treated mica imaged in 3 mM Tris buffer.

functionality of thesample. Several interactions havebeen exploited for CFM including friction, adhesion, energy dissipation, and biomolecular recognition. ${ }^{21}$ The double layer interactions of FEFM are sensitive only to charge density and, thus, are less specific than those possible with CFM. However, FEFM data are more easily interpreted because double layer interactions are wellunderstood, 22 and, significantly, FE FM does not require a functionalized tip. The two systems imaged here, fluidphase-supported bilayer membranes (F igure2) and single $\lambda$-DNA molecules (Figure 5), emphasize the high sensitivity and the nondestructive nature of this new charge mapping method. These results point to applications in biological imaging, such as mapping binding sites in large proteins or observing the distributions of charged species in biomembranes.

(21) Takano, H.; Kenseth, J . R.; Wong, S.-S.; O'Brien, J .; Porter, M D. Chem. Rev. 1999, 99, 2485-2890.

(22) Israelachvili, J . Intermolecular \& Surface Forces, 2nd ed.; Academic Press: San Diego, 1992; p 450.

\section{Conclusion}

FEFM maps nanometer-scale charge density in electrolyte solutions on the basis of the electric double layer interactions between the tip and sample. Because it is based on the lift mode, FEFM provides high-resolution images at typical AFM imaging rates and avoids topographic convolution. It can be applied to soft, biological materials such as fluid-phase-supported bilayer membranes and DNA. As a result of its simpleimplementation and clear interpretation, FEFM should be broadly applicable in many areas where fluid AFM imaging is of interest.

Acknowledgment. This work was supported by the Arnold and Mabel Beckman Foundation and the NSF Center for Biological and Environmental Nanotechnol ogy (NSF grant EEC-0118007).

LA035255F 